\title{
Simultaneous Quantification of Ten Active Components in Traditional Chinese Formula Sijunzi Decoction Using a UPLC-PDA Method
}

\author{
Kang An, ${ }^{1,2,3}$ Guo Jin-rui, ${ }^{1}$ Zhang Zhen, ${ }^{2}$ and Wang Xiao-long ${ }^{1}$ \\ ${ }^{1}$ School of Pharmacy, Nanjing University of Chinese Medicine, 138 Xianlin Road, Nanjing 210046, China \\ ${ }^{2}$ Discipline of Chinese and Western Integrative Medicine, Nanjing University of Chinese Medicine, 138 Xianlin Road, \\ Nanjing 210046, China \\ ${ }^{3}$ State Key Laboratory of Natural Medicines, China Pharmaceutical University, Nanjing 210009, China
}

Correspondence should be addressed to Wang Xiao-long; x.greg.wang@gmail.com

Received 25 September 2013; Accepted 17 April 2014; Published 20 May 2014

Academic Editor: Xiu-Ping Yan

Copyright (C) 2014 Kang An et al. This is an open access article distributed under the Creative Commons Attribution License, which permits unrestricted use, distribution, and reproduction in any medium, provided the original work is properly cited.

\begin{abstract}
Sijunzi decoction (SJZT), a traditional Chinese formula (TCMF) consisting of four herbs, has been widely used for the treatment of various gastrointestinal symptoms. However, its modernization process is hindered by the lack of a powerful quality control method that covers the major active components in the formula. The aim of this study was to establish a UPLC method for the quantitative determination of ten active components in Sijunzi decoction including ginsenoside $\mathrm{Rg}_{1}, \mathrm{Re}, \mathrm{Rb} \mathrm{b}_{1}$, liquiritin, liquiritigenin, glycyrrhizic acid, atractylenolide I, atractylenolide II, atractylenolide III, and pachymic acid. Separation was achieved using an ACQUITY UPLC BEHC ${ }_{18}$ column $(2.1 \mathrm{~mm} \times 100 \mathrm{~mm}, 1.7 \mu \mathrm{m})$ with a gradient elution program consisting of acetonitrile and $0.1 \%$ phosphoric acid solution. The detection wavelengths were set at 203, 254, 222, and $267 \mathrm{~nm}$. The method was validated for linearity, accuracy, precision, limit of detection, and limit of quantification. The validated method was successfully applied to the simultaneous quantification of ten active compounds from several finished batches of SJZT. This validated that UPLC method is expected to provide a new basis for the quality control of SJZT.
\end{abstract}

\section{Introduction}

Traditional Chinese herbal formulation (TCMF) has been widely used in the clinic for its well-proven efficacy with few side effects. Sijunzi decoction (SJZT) is one of the most famous TCMFs consisting of four herbs: Radix Ginseng, Poria cocos, Rhizoma Atractylodis Macrocephalae, and Radix Glycyrrhizae. In China, SJZT has long been used for the treatment of gastrointestinal disorders such as chronic gastritis and gastric and duodenal ulcer, and it could effectively attenuate nausea, vomiting, and diarrhea [1]. Clinical studies show that SJZT could effectively restore the homeostasis of the digestive tract in patients [2]. More recently, SJZT has been shown to ameliorate the intestinal flora disturbance in rat models of spleen deficiency syndrome [3]. Moreover, emerging evidences are showing that SJZT and modified
SJZT could play a good supporting role in suppressing tumors and confer a protective effect on gastrointestinal mucosa damage induced by chemotherapy [4].

Supporting these well-confirmed pharmacological efficacies, recent years have seen an increasing knowledge of the chemical components from SJZT. HPLC-MS was exploited to analyze the major components of SJZT, and eight ginsenosides (ginsenosides $\mathrm{Rg}_{1}$, Re, Rf, Ro, $\mathrm{Rb}_{1}, \mathrm{Rc}, \mathrm{Rb}_{2}$, and $\mathrm{Rd}$ ) and glycyrrhizic acid were identified through structural elucidation [1]. Recently, by employing the UPLC-Q-TOFMS technique, 66 phytochemical compounds were detected in Sijunzi decoction formula and 58 of them including ginsenosides, flavonoids, triterpenoid, and coumarins were tentatively identified by comparing the accurate mass and fragment information with the correlative references data [5]. It should be noted that the constituents and contents of the 
main active components existing in SJZT may be influenced by harvest time, plant origin, and manufacturing procedures, which could significantly affect the pharmacological effects and necessitate the quality assessment of SJZT. Undoubtedly, it is not easy to simultaneously determine all the components existing in the formula. However, simultaneous analysis of the main active components may be one possible solution. Previous studies have quantitatively determined the main components existing in the four individual herbs of SJZT, namely, Radix Ginseng [6, 7], Poria cocos [8], Rhizoma Atractylodis Macrocephalae[9, 10], and Glycyrrhiza uralensis [11-13], by using HPLC or UPLC methods. However, a satisfactory quantitative method of the major active components in SJZT for quality control purposes is not available. Simultaneous analysis for the main active compounds in each herb of SJZT has been suggested as one possible solution.

The aim of this research was to develop a convenient, reliable, and sensitive analytical method to determine the quantity of major compounds in SJZT by using ultraperformance liquid chromatography (UPLC). Specifically, ginsenoside $\mathrm{Rg}_{1}$, $\mathrm{Re}, \mathrm{Rb}_{1}$, liquiritin, liquiritigenin, glycyrrhizic acid, atractylenolide I, atractylenolide II, atractylenolide III, and pachymic acid were selected as the marker constituents for the relatively high contents in the individual herbs and their validated pharmacological effects, such as antiinflammation, brain protection effects, antioxidation effect, and hypoglycemic effect $[1,5]$. The potential application of this study could not only support a quality control of SJZT but also provide a theoretical basis for further in-depth research of SJZT in clinical research.

\section{Experimental}

2.1. Reagents and Chemicals. The four crude herbs, Radix Ginseng, Poria cocos, Rhizoma Atractylodis Macrocephalae, and Glycyrrhizae uralensis, were purchased from Nanjing Traffic Hospital (Nanjing, China). All samples were identified by one of the authors (Professor Wang Xiao-Long) as authentic herbal medicine. Ginsenoside $\mathrm{Rg}_{1}$, $\mathrm{Re}$, and $\mathrm{Rb}_{1}$ were purchased from Jilin University (Changchun, China); liquiritin, liquiritigenin, and glycyrrhizic acid were purchased from Chinese Food and Drug Inspection Institute; Atractylenolide III, Atractylenolide I, Atractylenolide II, and pachymic acid were purchased from Sichuan Weikeqi Biological Co., Ltd (Chengdu, China). The ten compounds used in the analysis were of analytical grade and their purity was more than $98 \%$. Their chemical structures are shown in Figure 1. Acetonitrile and methanol (HPLC grade) were purchased from Fisher Scientific (Waltham, MA, USA); phosphoric acid (analytical grade) was purchased from Nanjing Chemical Regents Company (Nanjing, China); water was purified by a Millipore Milli-Q system (Millipore, MA, USA); other reagents and chemicals were all obtained from various commercial sources and were of analytical grade.

2.2. Chromatographic Analysis. The analytes were separated on an ACQUITY UPLC H-class system with a PDA detector using $2.1 \mathrm{~mm} \times 100 \mathrm{~mm} \times 1.7 \mu \mathrm{m}$ ACQUITY BEH C18 column with a flow rate of $0.3 \mathrm{~mL} \mathrm{~min}^{-1}$. Separation was achieved using a gradient method. Acetonitrile (A) and $0.1 \%$ phosphoric acid (B) were selected and the gradient solvent system was as follows: $0-5 \mathrm{~min}$, (A) $19 \%$; 5-13 $\mathrm{min}, 19 \%-$ 30\%; 13-25 min, 30\%-50\%; 25-33 min, 50\%-60\%; 33-37 min, (A) $60 \%-68 \%$; 37-39 $\mathrm{min}$, (A) $68 \%-19 \%$; and $30-40 \mathrm{~min}$, (A) $19 \%$. The injection volume of samples was $5 \mu \mathrm{L}$ and temperature of the column oven was maintained at $30^{\circ} \mathrm{C}$. The UV wavelength was set at $203 \mathrm{~nm}$ (for ginsenoside $\mathrm{Rg}_{1}$, $\mathrm{Re}$, and $\mathrm{Rb}_{1}$ ), $254 \mathrm{~nm}$ (for liquiritin, glycyrrhizic acid, and liquiritigenin), $222 \mathrm{~nm}$ (for atractylenolide I and III), $276 \mathrm{~nm}$ (for atractylenolide II), and $242 \mathrm{~nm}$ (for pachymic acid).

2.3. Preparation of SJZT Samples. According to the original composition of SJZT, the four constituting herbs including Radix Ginseng (100 g), Poria cocos (100 g), Rhizoma Atractylodis Macrocephalae(100 g), and Glycyrrhizae uralensis (50 g) were crushed into small pieces and then mixed and decocted twice in $3500 \mathrm{~mL}$ water for $1 \mathrm{~h}$ in a glass flask. The decoction was filtered through 8 layers of gauze; the filtrate was concentrated in vacuum at $60^{\circ} \mathrm{C}$ at a final concentration of $2 \mathrm{~g} / \mathrm{mL}$.

Ethanol was added to an aliquot of $5 \mathrm{~mL}$ SJZT overnight in order to remove the polysaccharides. The supernatant was transferred into a test tube and evaporated to dryness with vacuum at room temperature. Finally, the residue was reconstituted in $5 \mathrm{~mL}$ methanol by vortex mixing for $5 \mathrm{~min}$ and centrifuged at $16,000 \mathrm{rpm}$ for 10 minutes. $5 \mu \mathrm{L}$ supernatant was injected into chromatographic systems for analysis.

2.4. Preparation of Standard Solutions. The standard stock solutions of ginsenoside $\operatorname{Rg}_{1}(300.00 \mu \mathrm{g} / \mathrm{mL})$, ginsenoside $\mathrm{Re}$ $(302.10 \mu \mathrm{g} / \mathrm{mL})$, ginsenoside $\mathrm{Rb}_{1}(300.00 \mu \mathrm{g} / \mathrm{mL})$, liquiritin $(90.30 \mu \mathrm{g} / \mathrm{mL})$, liquiritigenin $(87.00 \mu \mathrm{g} / \mathrm{mL})$, glycyrrhizic acid $(110.00 \mu \mathrm{g} / \mathrm{mL})$, atractylenolide III $(59.00 \mu \mathrm{g} / \mathrm{mL})$, atractylenolide I $(71.40 \mu \mathrm{g} / \mathrm{mL})$, atractylenolide II $(50.60 \mu \mathrm{g} / \mathrm{mL})$, and pachymic acid $(200.00 \mu \mathrm{g} / \mathrm{mL})$ were prepared in methanol. These solutions were stored at $4^{\circ} \mathrm{C}$ and were stable for at least 1 month.

2.5. Preparation of Negative Control Samples of SJZT. The negative control samples of SJZT were prepared by deriving one herb from the prescriptions. The herbs were accurately weighed according to the prescription of SJZT and prepared with the same procedure as for the sample preparation.

2.6. Method Validation. Specificity, linearity, limit of detection (LOD), limit of quantification (LOQ), precision (repeatability and intra- and interassay), and accuracy (recovery) of this UPLC method were evaluated in accordance with International Conference on Harmonization (ICH) [14].

The standard calibration curve for the linearity assay was prepared with seven different concentrations of diluted standard solutions (ginsenoside $\mathrm{Rg}_{1}$, ginsenoside $\mathrm{Re}$, ginsenoside $\mathrm{Rb}_{1}$, liquiritin, glycyrrhizic acid, liquiritigenin, atractylenolide I, atractylenolide II, atractylenolide III, and 


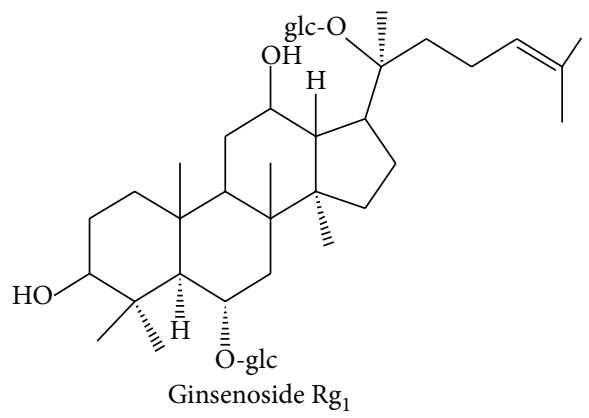<smiles>O=C1CC(c2ccc(O)cc2)Oc2cc(O)ccc21</smiles><smiles>COc1ccc(C2CC(=O)c3ccc(O)cc3O2)cc1</smiles>

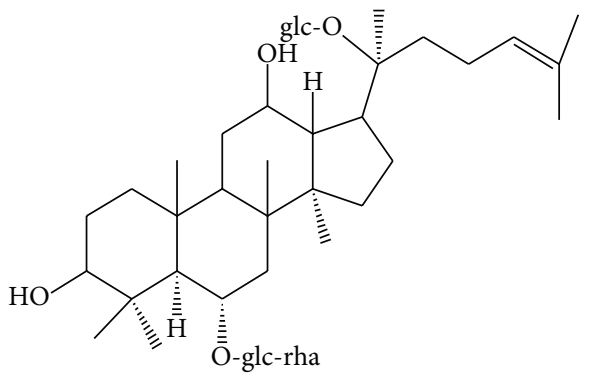<smiles></smiles>

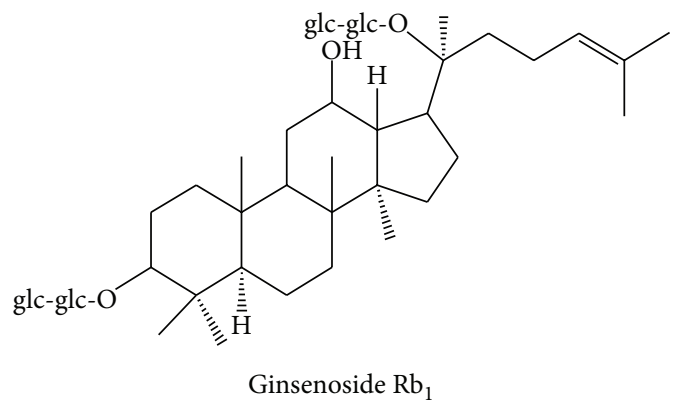<smiles>C=C(C)CCC(C(=O)O)[C@]1(C)C(O)C[C@]2(C)C3=C(CC[C@]21C)[C@@]1(C)CCC(OC(C)=O)C(C)(C)[C@@]1(O)CC3</smiles><smiles>C=C1CCCC2(C)CC3OC(=O)C(C)=C3C[C@H]12</smiles>

Atractylenolide I<smiles>C=C1CCCC2(C)CC3(O)OC(=O)C(C)=C3C[C@H]12</smiles>

Atractylenolide III<smiles>C=C1CCCC2(C)C=C3OC(=O)C(C)=C3C[C@H]12</smiles>

Atractylenolide II

FIGURE 1: The chemical structure of ginsenoside $\mathrm{Rg}_{1}, \mathrm{Re}, \mathrm{Rb}_{1}$, liquiritin, liquiritigenin, glycyrrhizic acid, atractylenolide I, atractylenolide II, atractylenolide III, and pachymic acid.

pachymic acid). The lower limit of quantification (LLOQ) was determined as the lowest concentration point of the standard curve and the signal-to-noise ratio was higher than 10 . The lower limit of detection (LLOD) was defined as the amount that could be detected with a signal-to-noise ratio of 3 .

The precision of the analytical method was evaluated by intrabatch and interbatch variability. Three different concentrations of standards (low, medium, and high) were prepared. The quantity of each component was determined by the respective calibration curve. RSD was used to measure precision. The interbatch reproducibility test was carried out on three different batches.

Recovery studies were carried out by spiking three concentrations of mixed standards at low (50\% of the known 

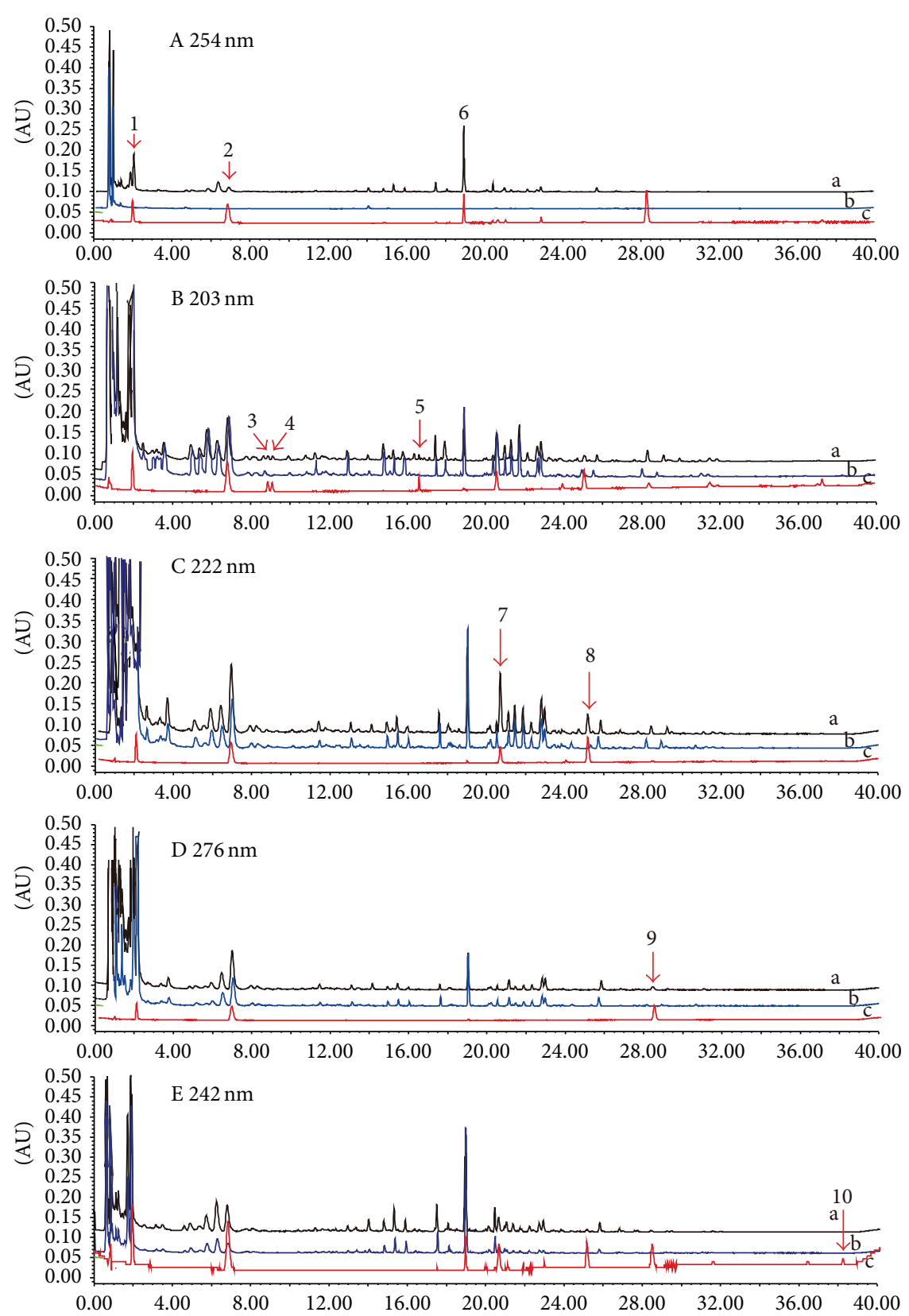

FIGURE 2: Ultraperformance liquid chromatography (UPLC) chromatograms at different wavelengths of standard mixture (a), SJZT extraction (b), and negative control samples (c). Peaks: (1) liquiritin, (2) liquiritigenin, (3) ginsenoside $\mathrm{Rg}_{1}$, (4) ginsenoside Re, (5) ginsenoside $\mathrm{Rb}_{1}$, (6) glycyrrhizic acid, (7) atractylenolide III, (8) atractylenolide I, (9) atractylenolide II, and (10) pachymic acid.

amounts), medium (100\% of the known amounts), and high (200\% of the known amounts) in the $5 \mathrm{~mL}$ of SJZT. Then, the spiked samples were then extracted, processed, and quantified in accordance with the methods mentioned above.

\section{Results and Discussion}

3.1. Optimization of the UPLC Conditions. Optimization of the separation conditions for HPLC analysis was performed including the mobile phase composition, gradient elution program, and wavelength. To obtain chromatograms with better resolution of adjacent peaks within shorter time, the chromatographic conditions were optimized. Methanol and acetonitrile were compared in the experiment. The result showed that acetonitrile was much better as it could result in a better resolution and shorter time for analysis. In addition, water and $0.1 \%$ phosphoric acid/water were investigated and the result showed that $0.1 \%$ phosphoric acid/water was better than water. As a result ACQUITY UPLC BEH $\mathrm{C}_{18}$ column $(2.1 \mathrm{~mm} \times 100 \mathrm{~mm}, 1.7 \mu \mathrm{m})$ with acetonitrile and 
TABLE 1: The linear regression data, LODs, and LOQs of ten compounds.

\begin{tabular}{|c|c|c|c|c|c|}
\hline Components & Regression equations & $R^{2}$ & $\begin{array}{c}\text { Linear range } \\
(\mu \mathrm{g} / \mathrm{mL})\end{array}$ & $\begin{array}{l}\text { LODs } \\
(\mu \mathrm{g} / \mathrm{mL})\end{array}$ & $\begin{array}{l}\text { LOQs } \\
(\mu \mathrm{g} / \mathrm{mL})\end{array}$ \\
\hline Ginsenoside $\mathrm{Rg}_{1}$ & $y=2123.1 x+1493.3$ & 1 & $4.68-300.00$ & 0.07 & 0.23 \\
\hline Ginsenoside Re & $y=1701.3 x+2520.7$ & 0.9998 & $4.72-302.10$ & 0.07 & 0.23 \\
\hline Ginsenoside $\mathrm{Rb}_{1}$ & $y=1465 x+2077.4$ & 0.9998 & $4.68-300.00$ & 0.06 & 0.20 \\
\hline Liquiritin & $y=4334.7 x+1378.8$ & 0.9998 & $1.41-90.30$ & 0.02 & 0.07 \\
\hline Liquiritigenin & $y=7817.1 x+3469.8$ & 0.9998 & $1.36-87.00$ & 0.02 & 0.07 \\
\hline Glycyrrhizic acid & $y=3181.9 x+1888$ & 0.9998 & $1.72-110.00$ & 0.03 & 0.10 \\
\hline Atractylenolide III & $y=34419 x+6432.6$ & 0.9999 & $0.92-59.00$ & 0.01 & 0.03 \\
\hline Atractylenolide I & $y=18610 x+8178.9$ & 0.9998 & $1.12-71.40$ & 0.02 & 0.07 \\
\hline Atractylenolide II & $y=40016 x+9753.9$ & 0.9999 & $0.79-50.60$ & 0.01 & 0.03 \\
\hline Pachymic acid & $y=2060.1 x+3937.9$ & 0.9996 & $3.13-200.00$ & 0.05 & 0.16 \\
\hline
\end{tabular}

$y=A x+B ; y$ is peak area; $x$ is concentration of the analytes $(\mu \mathrm{g} / \mathrm{mL}) ; r$ is the correlation coefficient of the equation.

TABLE 2: Precision, repeatability, and stability of ten compounds in SJZT $(n=6)$.

\begin{tabular}{|c|c|c|c|c|c|}
\hline \multirow{2}{*}{ Analytes } & \multicolumn{3}{|c|}{ Precision } & \multirow{2}{*}{ Repeatability RSD (\%) } & \multirow{2}{*}{ Stability RSD (\%) } \\
\hline & Levels (ug/mL) & Intraday RSD (\%) & Interday RSD (\%) & & \\
\hline \multirow{3}{*}{ Ginsenoside $\mathrm{Rg}_{1}$} & 7.03 & $1.05 \%$ & $0.29 \%$ & \multirow{3}{*}{$0.98 \%$} & \multirow{3}{*}{$1.01 \%$} \\
\hline & 37.50 & $0.30 \%$ & $0.20 \%$ & & \\
\hline & 240.00 & $0.85 \%$ & $1.04 \%$ & & \\
\hline \multirow{3}{*}{ Ginsenoside Re } & 7.08 & $0.60 \%$ & $0.22 \%$ & \multirow{3}{*}{$0.87 \%$} & \multirow{3}{*}{$1.04 \%$} \\
\hline & 37.76 & $0.36 \%$ & $0.42 \%$ & & \\
\hline & 241.68 & $0.81 \%$ & $0.93 \%$ & & \\
\hline \multirow{3}{*}{ Ginsenoside $\mathrm{Rb}_{1}$} & 7.03 & $0.28 \%$ & $0.22 \%$ & \multirow{3}{*}{$1.20 \%$} & \multirow{3}{*}{$0.92 \%$} \\
\hline & 37.50 & $0.17 \%$ & $0.16 \%$ & & \\
\hline & 240.00 & $1.15 \%$ & $0.98 \%$ & & \\
\hline \multirow{3}{*}{ Liquiritin } & 2.12 & $0.33 \%$ & $0.70 \%$ & \multirow{3}{*}{$0.48 \%$} & \multirow{3}{*}{$0.57 \%$} \\
\hline & 11.29 & $0.54 \%$ & $0.35 \%$ & & \\
\hline & 72.24 & $1.18 \%$ & $1.03 \%$ & & \\
\hline \multirow{3}{*}{ Liquiritigenin } & 2.04 & $0.83 \%$ & $0.70 \%$ & \multirow{3}{*}{$0.27 \%$} & \multirow{3}{*}{$0.92 \%$} \\
\hline & 10.88 & $0.39 \%$ & $0.49 \%$ & & \\
\hline & 69.60 & $0.39 \%$ & $0.31 \%$ & & \\
\hline \multirow{3}{*}{ Glycyrrhizic acid } & 2.58 & $0.91 \%$ & $1.04 \%$ & \multirow{3}{*}{$0.28 \%$} & \multirow{3}{*}{$0.73 \%$} \\
\hline & 13.75 & $0.21 \%$ & $1.13 \%$ & & \\
\hline & 88.00 & $0.71 \%$ & $0.72 \%$ & & \\
\hline \multirow{3}{*}{ Atractylenolide III } & 1.38 & $1.08 \%$ & $0.46 \%$ & \multirow{3}{*}{$0.63 \%$} & \multirow{3}{*}{$0.84 \%$} \\
\hline & 7.38 & $0.96 \%$ & $0.50 \%$ & & \\
\hline & 47.20 & $0.93 \%$ & $0.83 \%$ & & \\
\hline \multirow{3}{*}{ Atractylenolide I } & 1.67 & $1.03 \%$ & $0.58 \%$ & \multirow{3}{*}{$0.81 \%$} & \multirow{3}{*}{$0.92 \%$} \\
\hline & 8.93 & $1.07 \%$ & $0.97 \%$ & & \\
\hline & 57.12 & $0.87 \%$ & $0.72 \%$ & & \\
\hline \multirow{3}{*}{ Atractylenolide II } & 1.19 & $1.03 \%$ & $0.64 \%$ & & \\
\hline & 6.33 & $0.63 \%$ & $0.62 \%$ & & \\
\hline & 40.48 & $0.97 \%$ & $0.72 \%$ & & \\
\hline \multirow{3}{*}{ Pachymic acid } & 4.69 & $0.52 \%$ & $0.48 \%$ & & \\
\hline & 25.00 & $0.97 \%$ & $1.10 \%$ & & \\
\hline & 160.00 & $0.61 \%$ & $0.37 \%$ & & \\
\hline
\end{tabular}

RSD: relative standard deviation. 
TABLE 3: Recovery of eight components in Sijunzi $(n=3)$.

\begin{tabular}{|c|c|c|c|c|c|c|}
\hline Components & Contents (ug/mL) & $\begin{array}{c}\text { Quantity added } \\
\text { (ug/mL) }\end{array}$ & $\begin{array}{l}\text { Theoretical amount } \\
\text { (ug/mL) }\end{array}$ & Recorded amount (ug/mL) & Recovery (\%) & $\operatorname{RSD}(\%)$ \\
\hline \multirow{3}{*}{ Ginsenoside $\mathrm{Rg}_{1}$} & 64.83 & 37.5 & 102.33 & 102.42 & 100.25 & 0.65 \\
\hline & 64.83 & 75 & 139.83 & 139.44 & 99.48 & 0.78 \\
\hline & 64.83 & 150 & 214.83 & 210.05 & 96.81 & 0.54 \\
\hline \multirow{3}{*}{ Ginsenoside $\mathrm{Rb}_{1}$} & 48.17 & 37.5 & 85.67 & 85.24 & 98.85 & 0.67 \\
\hline & 48.17 & 75 & 123.17 & 119.96 & 95.72 & 0.82 \\
\hline & 48.17 & 150 & 198.17 & 191.81 & 95.76 & 0.87 \\
\hline \multirow{3}{*}{ Ginsenoside Re } & 61.63 & 18.88 & 80.51 & 80.95 & 102.33 & 0.53 \\
\hline & 61.63 & 37.76 & 99.39 & 99.73 & 100.9 & 0.20 \\
\hline & 61.63 & 75.53 & 137.16 & 137.13 & 99.96 & 0.26 \\
\hline \multirow{3}{*}{ Liquiritin } & 57.39 & 22.58 & 79.97 & 78.98 & 95.62 & 0.76 \\
\hline & 57.39 & 45.15 & 102.54 & 100.78 & 96.1 & 0.87 \\
\hline & 57.39 & 90.3 & 147.69 & 143.72 & 95.6 & 0.84 \\
\hline \multirow{3}{*}{ Liquiritigenin } & 47.48 & 21.75 & 69.23 & 69.27 & 100.18 & 0.25 \\
\hline & 47.48 & 43.5 & 90.98 & 91.67 & 101.59 & 0.79 \\
\hline & 47.48 & 87 & 134.48 & 136.8 & 102.67 & 0.89 \\
\hline \multirow{3}{*}{ Glycyrrhizic acid } & 111.18 & 55.00 & 166.18 & 164.12 & 96.25 & 0.62 \\
\hline & 111.18 & 110.00 & 221.18 & 217.71 & 96.84 & 0.63 \\
\hline & 111.18 & 220.00 & 331.18 & 330.52 & 99.7 & 0.36 \\
\hline \multirow{3}{*}{ Atractylenolide III } & 42.08 & 29.50 & 71.58 & 72.45 & 102.95 & 0.83 \\
\hline & 42.08 & 59.00 & 101.08 & 100.81 & 99.54 & 0.30 \\
\hline & 42.08 & 118.00 & 160.08 & 160.29 & 100.18 & 0.24 \\
\hline \multirow{3}{*}{ Atractylenolide I } & 10.50 & 8.93 & 19.43 & 19.37 & 99.33 & 0.21 \\
\hline & 10.50 & 17.85 & 28.35 & 28.47 & 100.67 & 0.20 \\
\hline & 10.50 & 37.50 & 48.00 & 46.15 & 95.07 & 0.62 \\
\hline
\end{tabular}

Recovery $(\%)=($ recorded amount - original amount $) /$ spiked amount $* 100 \%$.

$0.1 \%$ phosphoric acid/water was selected as the preferred chromatographic conditions.

Moreover, different gradient profiles were also optimized. Actually, we tried to simplify the gradient elution system and shorten the analysis time, but peaks for atractylenolide III and atractylenolide I have not been completely separated except for the current condition to in the gradient program mentioned above.

In this experiment, the specificity of UV absorption was also investigated, using the present chromatographic conditions and comparing a SJZT sample with a standard mixture. The UV absorbance and the best UV detection wavelength of each compound in SJZT were confirmed as follows: ginsenoside $\mathrm{Rg}_{1}$, $\mathrm{Re}$, and $\mathrm{Rb}_{1}(203 \mathrm{~nm})$, liquiritin, glycyrrhizic acid, and liquiritigenin $(254 \mathrm{~nm})$, atractylenolide I and atractylenolide III $(222 \mathrm{~nm})$, atractylenolide II $(276 \mathrm{~nm})$, and pachymic acid $(242 \mathrm{~nm})$.

3.2. Validation of the UPLC Method. The validation study allowed the evaluation of the method for its suitability for routine analysis.
3.2.1. Specificity. Representative chromatograms of the standard solution, sample solution, and negative control samples at different UV wavelength were shown in Figure 2. The chromatographic peaks were identified by comparing their retention time with that of each reference compound. In addition, chromatograms of the negative control samples further confirmed the specificity of this method.

3.2.2. Calibration Curves, LODs, and LOQs. The linearity of the developed method was assessed using seven different concentrations each of ginsenoside $\mathrm{Rg}_{1}$, ginsenoside $\mathrm{Re}$, ginsenoside $\mathrm{Rb}_{1}$, liquiritin, glycyrrhizic acid, liquiritigenin, atractylenolide I, atractylenolide II, atractylenolide III, and pachymic acid and the observed concentrated ranges were as follows: 4.68 300.00, 4.72 302.10, 4.68 300.00, 1.41 90.30, $1.72 \sim 110.00,1.36 \sim 87.00,1.12 \sim 71.40,0.79 \sim 50.60,0.92 \sim 59.00$, and $3.13 \sim 200.00 \mu \mathrm{g} / \mathrm{mL}$, respectively. The correlation coefficient $\left(R^{2}\right)$ of all biomarkers has good linearity of over $>$ 0.9996 in the aforementioned ranges. The LODs and LOQs of the ten analytes were $0.01 \sim 0.07$ and $0.03 \sim 0.23 \mu \mathrm{g} / \mathrm{mL}$, respectively (Table 1). 
TABLE 4: Contents of eight components in three batches of SJZT.

\begin{tabular}{|c|c|c|c|c|c|c|c|c|c|}
\hline \multirow[b]{2}{*}{ Sample } & \multirow[b]{2}{*}{ Source } & \multicolumn{8}{|c|}{ Contents (mg/g) } \\
\hline & & $\begin{array}{c}\text { Ginsenoside } \\
\mathrm{Rg}_{1}\end{array}$ & $\begin{array}{c}\text { Ginsenoside } \\
\text { Re }\end{array}$ & $\begin{array}{c}\text { Ginsenoside } \\
\mathrm{Rb}_{1}\end{array}$ & Liquiritin & Liquiritigenin & $\begin{array}{c}\text { Glycyrrhizic } \\
\text { acid }\end{array}$ & $\begin{array}{c}\text { Atractylenolide } \\
\text { III }\end{array}$ & Atractylenolide I \\
\hline 1 & Batch 1 & 0.254 & 0.115 & 0.184 & 0.659 & 0.078 & 0.258 & 0.020 & 0.013 \\
\hline 2 & Batch 2 & 0.445 & 0.185 & 0.261 & 0.858 & 0.100 & 0.345 & 0.043 & 0.017 \\
\hline 3 & Batch 3 & 0.209 & 0.094 & 0.116 & 0.600 & 0.067 & 0.572 & 0.039 & 0.011 \\
\hline
\end{tabular}

3.2.3. Precision, Repeatability, and Stability. Intraday and interday variations were chosen to determine the precision of the developed assay. The analyzed data showed that relative standard deviation (RSD) of intra- and interday was in the range of $0.21-1.18 \%$ and $0.20-1.10 \%(n=6)$, the RSD of the SJZT repeatability, and stability test was in the range of $0.20-$ $0.89 \%$ and $0.57 \%-1.04 \%$, respectively (Table 2 ). None of the precision, repeatability, and stability data exceeded 5\%, except atractylenolide II and pachymic acid (the content of those two components were lower than the LODs).

3.2.4. Accuracy. The accuracy of the method was assessed by a recovery assay. The spiked samples were then extracted, processed, and quantified in accordance with the methods mentioned above. The measured data showed that the recovery of the investigated components ranged from $95.07 \%$ to $102.67 \%$, and their RSD values were all less than $3.0 \%$ (Table 3). Recovery data represented the accuracy of the method and is sufficient for usual analysis

3.2.5. Applications. The established analytical method was subsequently applied for the simultaneous determination of the ten markers in 3 batches of SJZT. The results are presented in Table 4. The results showed that there are remarkable differences among the contents of the ten components in SJZT from the same or different batches.

\section{Conclusion}

In this study, a UPLC method for the simultaneous determination of ten active ingredients in SIZT has been developed and the results showed that it could be used for the quality control of the SJZT. Thus, this validated that UPLC method could be expected to provide a new basis for the quality control of SJZT.

\section{Conflict of Interests}

The authors declare that there is no conflict of interests regarding publication of this paper.

\section{Authors' Contribution}

Kang An and Guo Jin-rui contributed equally to this work.

\section{Acknowledgments}

This study was financially supported by the Youth Natural Science Fund of Nanjing University of Chinese Medicine (no. 11XZR09), National Natural Science Fund of China (no. 81202983), Open Project Program of State Key Laboratory of Natural Medicines, China Pharmaceutical University (no. SKLNMKF201209), and the Priority Academic Program Development of Jiangsu Higher Education Institutions (PAPD).

\section{References}

[1] Y. Liu, J. Yang, and Z. Cai, "Chemical investigation on Sijunzi decoction and its two major herbs Panax ginseng and Glycyrrhiza uralensis by LC/MS/MS," Journal of Pharmaceutical and Biomedical Analysis, vol. 41, no. 5, pp. 1642-1647, 2006.

[2] J. H. Guo, G. Chen, S. Q. Yang, M. H. Wei, and X. Chen, "Clinical observation of the role of Chenxia Sijunzi decoction in promoting the recovery of gastrointestinal function in critically ill patients," Zhongguo Wei Zhong Bing Ji Jiu Yi Xue, vol. 24, no. 11, pp. 674-676, 2012.

[3] Z. Wang, Y. Peng, and X. B. Li, "Effect of sijunzi decoction on the intestinal flora disturbance in two rat models of Pi-deficiency syndrome," Zhongguo Zhong Xi Yi Jie He Za Zhi, vol. 29, no. 9, pp. 825-829, 2009.

[4] C. Liang, S. H. Zhang, and Z. D. Cai, "Effects of early intestinal application of sijunzi decoction on immune function in postoperational patients of gastrointestinal tumor," Zhongguo Zhong Xi Yi Jie He Za Zhi, vol. 25, no. 12, pp. 1070-1073, 2005.

[5] Y. Wang, S. He, X. Cheng, Y. Lu, Y. Zou, and Q. Zhang, "UPLCQ-TOF-MS/MS fingerprinting of Traditional Chinese Formula SiJunZiTang," Journal of Pharmaceutical and Biomedical Analysis, vol. 80, pp. 24-33, 2013.

[6] P. N. Brown, R. Yu, T. Cain et al., "Determination of ginsenoside content in Panax ginseng C.A. Meyer and Panax quinquefolius L. root materials and finished products by high-performance liquid chromatography with ultraviolet absorbance detection: interlaboratory study," Journal of AOAC International, vol. 96, no. 1, pp. 12-19, 2013.

[7] X. Jin, L. Y. Zhu, H. Shen et al., "Influence of sulphur-fumigation on the quality of white ginseng: a quantitative evaluation of major ginsenosides by high performance liquid chromatography," Food Chemistry, vol. 135, no. 3, pp. 1141-1147, 2012.

[8] S. Che, Q. Li, Y.-S. Huo, X. H. Chen, and K. S. Bi, "RPHPLC simultaneous determination of five triterpenoid acids in different parts of Poria cocos by UV wavelengths switch," Yaoxue Xuebao, vol. 45, no. 4, pp. 494-497, 2010. 
[9] Q. Chen, H. He, P. Li, J. Zhu, and M. Xiong, "Identification and quantification of atractylenolide I and atractylenolide III in Rhizoma Atractylodes Macrocephala by liquid chromatographyion trap mass spectrometry," Biomedical Chromatography, vol. 27, no. 6, pp. 699-707, 2013.

[10] H. P. Chen, J. H. Zhang, X. Y. Wang, and L. Chen, "Contents comparision of atractylode I, II, III in rhizoma atractylodis macrocephalae and the processed with soils," Zhong Yao Cai, vol. 34, no. 3, pp. 354-357, 2011.

[11] Y. P. Wu, X. S. Meng, Y. R. Bao, S. Wang, and T. G. Kang, "Simultaneous quantitative determination of nine active chemical compositions in traditional Chinese medicine Glycyrrhiza by RP-HPLC with full-time five-wavelength fusion method," The American Journal of Chinese Medicine, vol. 41, no. 1, pp. 211219, 2013.

[12] Y. T. Kamal, M. Singh, E. T. Tamboli, R. Parveen, S. M. Zaidi, and S. Ahmad, "Rapid RP-HPLC method for the quantification of glabridin in crude drug and in polyherbal formulation," Journal of Chromatographic Science, vol. 50, no. 9, pp. 779-784, 2012.

[13] S. Gupta, R. Sharma, P. Pandotra, S. Jaglan, and A. P. Gupta, "Chromolithic method development, validation and system suitability analysis of ultra-sound assisted extraction of glycyrrhizic acid and glycyrrhetinic acid from Glycyrrhiza glabra," Natural Product Communications, vol. 7, no. 8, pp. 991-994, 2012.

[14] International conference of harmonization Q2B: Validation of analytical procedures-methodology. US FDA Federal Register, pp. 27463-27467, 1997. 

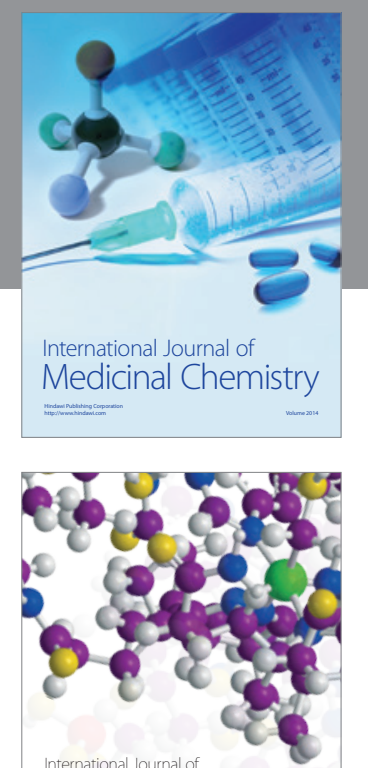

\section{Carbohydrate} Chemistry

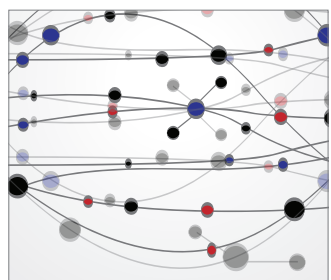

The Scientific World Journal
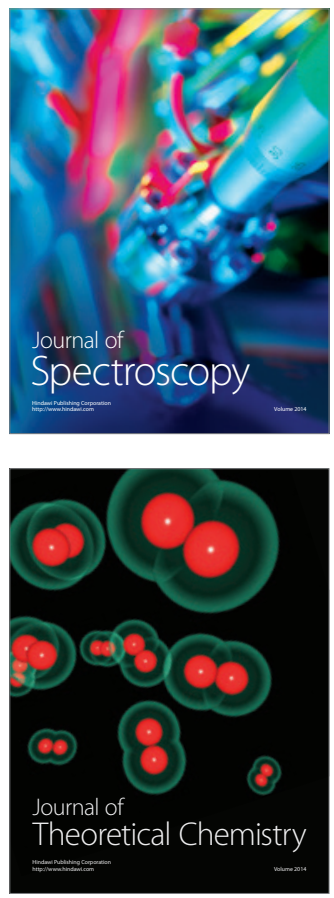
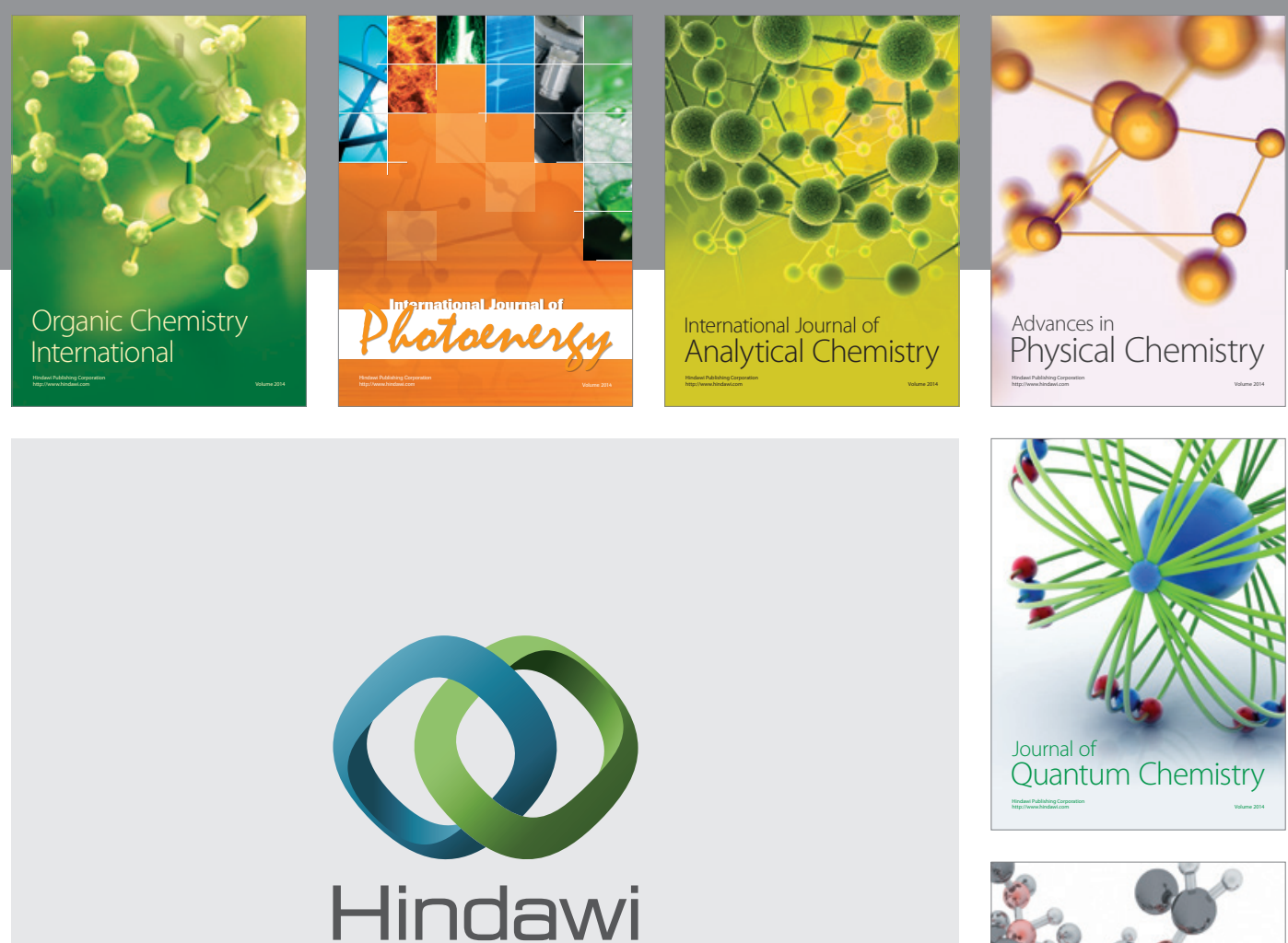

Submit your manuscripts at

http://www.hindawi.com

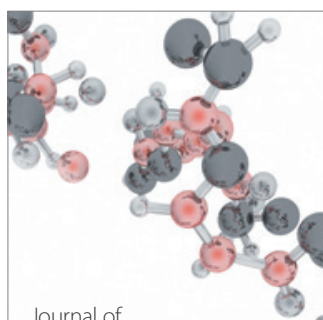

Analytical Methods

in Chemistry

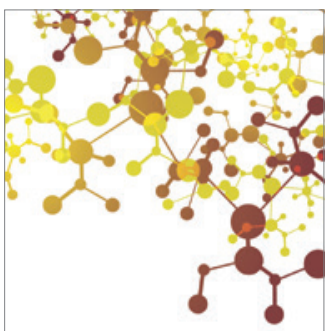

Journal of

Applied Chemistry

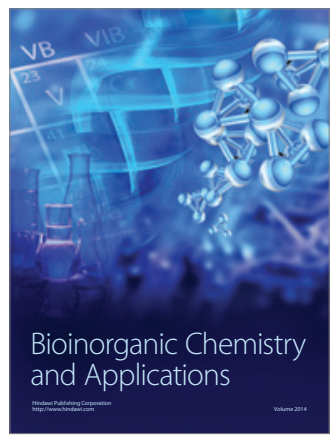

Inorganic Chemistry
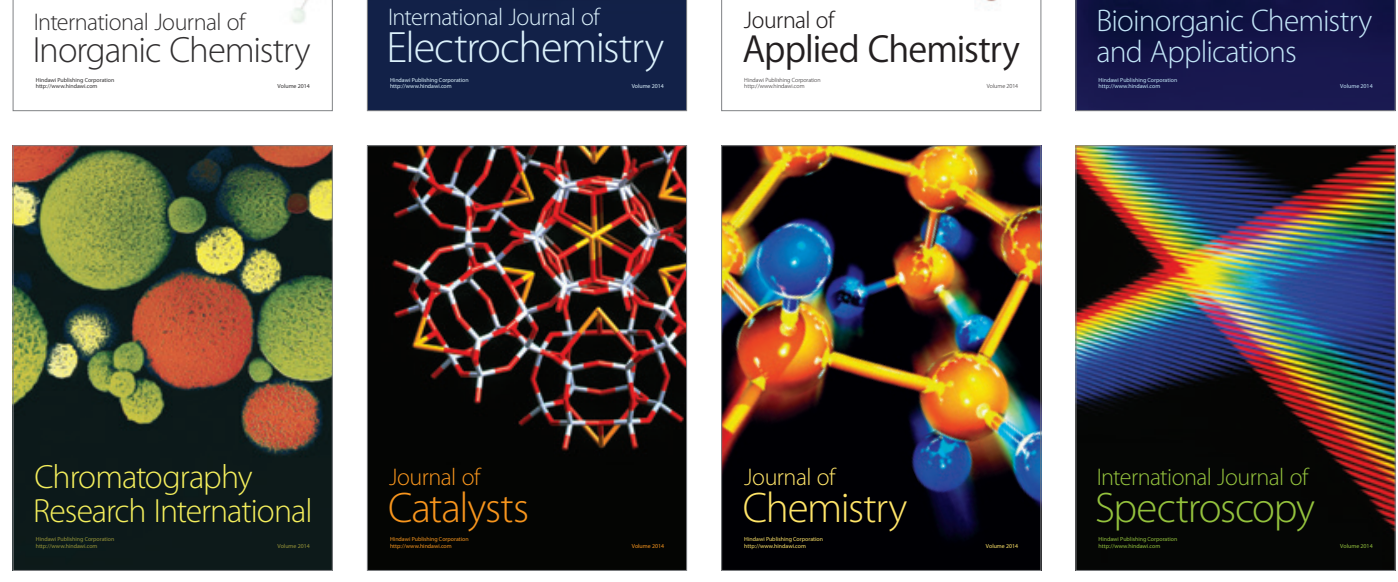